\title{
Theranostics
}

Editorial

2012; 2(1):122-124. doi: 10.7150/thno.4051

\section{Magnetic Nanoparticle-Based Theranostics}

\section{Jin Xie ${ }^{1 凶}$ and Sangyong Jon ${ }^{2 \bowtie}$}

1. Department of Chemistry and Bio-Imaging Research Center, University of Georgia, Athens, GA 30602, USA

2. Cell Dynamics Research Center, School of Life Sciences, Gwangju Institute of Science and Technology, 261 Chemdangwagi-ro, Gwangju 500-712, Republic of Korea.

$\triangle$ Corresponding author: jinxie@uga.edu and syjon@gist.ac.kr

(C) Ivyspring International Publisher. This is an open-access article distributed under the terms of the Creative Commons License (http://creativecommons.org/ licenses/by-nc-nd/3.0/). Reproduction is permitted for personal, noncommercial use, provided that the article is in whole, unmodified, and properly cited.

Received: 2012.01.05; Accepted: 2012.01.12; Published: 2012.01.16

\begin{abstract}
This theme issue provides a timely collection of studies on magnetic nanoparticle-based imaging, bio-sensing, therapy and/or their combinations.
\end{abstract}

Key words: Magnetic nanoparticles, biomedical imaging, biosensor, hyperthermia, gene/drug delivery

Magnetic nanoparticles have long been an important class of biomaterials. Iron oxide nanoparticles, for instance, have been used in the clinic as MR contrast probes, mostly for improving the visibility of lesions in the reticuloendothelial system (RES) organs, such as the liver and lymph nodes. Recently, a new set of chemistry has emerged, which allows one to prepare nanoparticles with fine control over a wide range of parameters, including size, shape, composition, magnetization, surface coating and surface charge, etc [1]. Such a transition did not only affect the applications of magnetic nanoparticles, but also opened many new avenues. The idea is to see the nanoparticles as not merely tiny magnetic crystals but rather, platforms with large surface-to-volume ratio. By harnessing the well-developed surface chemistry, one can load a wide range of functionalities onto the particle surface. These include biovectors--such as peptides, antibodies or aptamers--which are able to guide the migration of nanoparticles in a living subject and to accumulate them preferentially in the areas of interest, such as tumor. On top of that, the nanoplatform can be further loaded with imaging motifs or therapeutic agents, and as a consequence, to be upgraded to multifunctional nanogadgets with either multimodal imaging capabilities or theranostic features.
In detail, studies on magnetic nanoparticles are currently focused on the following subjects:

- Preparation of novel magnetic nanoparticle formulas with unique physical and surface properties.

- Studies on the toxicity of magnetic nanoparticles.

- In vitro detection of biomarkers that are of diagnostic and prognostic values using magnetic nanoparticles as the tags.

- Magnetic nanoparticle-based probes for cell tracking, MR imaging, and multimodal imaging.

- Magnetic nanoparticles-based therapeutics, such as for hyperthermia, drug delivery and gene delivery.

In this special issue, we invite scholars from worldwide to comment on or report cutting-edge studies on the preceding topics. It starts with an informative review written by Jon et al. on constructing magnetic nanoparticle-based, multifunctional theranostic systems [2]. This review discusses a broad spectrum of important topics in the field, including targeting strategies, conjugation techniques and surface engineering means used to shield nanoparticles from mononuclear phagocytic system. Subsequent to this is another review article contributed by Zhen and Xie, who summarize the current progress of using 
manganese-containing nanoparticles as MRI contrast agents [3]. Unlike iron oxide nanoparticles that are mostly used as $T_{2}$ contrast probes by causing hypointensities, manganese nanoparticles induce hyperintensities on $T_{1}$-weighted maps and therefore, can be diagnostically more favorable. It is also possible to load therapeutics onto these manganese nanoplatforms to upgrade them to theranostic agents. In addition to working as in vivo imaging contrast probes, magnetic nanoparticles have also found uses in in vitro biosensing. Here Shao et al. report their work on developing diagnostic magnetic resonance systems (DMR) to detect cellular biomarkers with high sensitivity and efficiency [4]. The DMR system has been dramatically improved in the past decade, thanks to the emergence of new magnetic nanoparticle biosensors, bioconjugation strategies, and highly sensitive miniaturized NMR systems. The system now enables parallel and rapid measurements from small sample volumes and on a wide range of targets, including whole cells, proteins, DNA/mRNA, metabolites, drugs, viruses and bacteria. Subsequently, we have two research articles. The first one comes from Ho et al., who studied tumor targeting with an iron oxide nanoparticle-tumstatin conjugate in a novel three-dimensional tissue culture model closely mimicking the in vivo tumor microenvironment [5]. Such a spheroid was constructed with a leaky endothelium coating and a glioma tumor mass core. The results showed that the tumstatin-iron oxide nanoparticle conjugates could penetrate and selectively target to endothelial cell coating. This spheroid platform may serve as an economic alternative to evaluate particle-tumor microenvironment interaction. In the second research article, $\mathrm{Li}$ et al. report a side-by-side comparison study on two types of iron oxide nanoparticles [6], i.e. thermally cross-linked superparamagnetic iron oxide nanoparticles (TCL-SPION) and monocrystalline iron oxide nanoparticles (MION-47). In particular, they incubated these two types of nanoparticles with murine macrophage cells RAW264.7 and evaluated the impact of the particles on the secretion of interlukin-6 (IL-6) and tumor necrosis factor-alpha (TNF- $\alpha$ ), the production of nitric oxides, and the mitochondrial membrane potentials. While no significant impact on cell viability and growth velocity was observed, it was found that the use of both kinds of nanoparticles led to increased secretion levels of IL-6 and TNF-a. In addition, MION-47 also led to the production of nitric oxides and more collapses of mitochondrial membrane potential. After these two research articles, Huang et al. discuss on how to improve MRI contrast and detection with engineered magnetic nanoparticles [7]. This work tries to shed some light on the relationship between the intrinsic parameters of magnetic nanoparticles (e.g. composition, size and surface properties as well as the degree of aggregation) and the MRI relaxivities that decide the performance of particles as imaging/diagnostic agents. Also discussed in this work are newly developed MRI methods which are able to improve the detection and quantification of the engineered magnetic nanoparticles. Magnetic nanoparticles can also be combined with other imaging agents to arrive at multimodality imaging probes. For instance, MRI/PET [8], MRI/optical [9] and MRI/PET/optical [10] imaging probes have been reported previously. In this special issue, Cai et al. overview the progress of constructing magnetic microbubbles [11]. Magnetic microbubbles are dual-modal imaging probes with both MR and ultrasound imaging functionalities. In addition, it is postulated that the embedded magnetic nanoparticles as well as other payloads can be delivered to desired regions and released in response to appropriate ultrasound exposure. Last, Zhao et al. report a study on iron oxide nanoparticle-mediated hyperthermia for the treatment of head and neck cancer [12]. It was found that temperature in nanoparticle-dosed tumor increased to $40{ }^{\circ} \mathrm{C} \sim 50{ }^{\circ} \mathrm{C}$ within 5 minutes by heating with an alternating magnetic field (AMF). Such a temperature increase fit well with theoretical simulation results. Further histology studies found that the heating-induced tumor cell death was mostly achieved through necrosis rather than apoptosis.

In summary, the use of magnetic nanoparticles in diagnosis and therapy is by all means a research hotspot and has attracted much attention. In this special issue, we have solicited 5 review articles and 3 research papers from experienced scholars in the field, with an aim of bringing broad and up-to-date knowledge to the audience. It is our hope that this timely issue will benefit students and researchers who are interested in this topic.

\section{Conflict of Interest}

The authors have declared that no conflict of interest exists.

\section{References}

1. Xie J, Liu G, Eden HS, Ai H, Chen X. Surface-engineered magnetic nanoparticle platforms for cancer imaging and therapy. Acc Chem Res. 2011; 44: 883-92.

2. Yu MK, Park J, Jon S. Targeting strategies for multifunctional nanoparticles in cancer imaging and therapy. Theranostics. 2012; 2: 3-44.

3. Zhen Z, Xie J. Development of manganese-based nanoparticles as contrast probes for magnetic resonance imaging. Theranostics. 2012; 2: 45-54. 
4. Shao H, Min C, Issadore D, Liong M, Yoon TJ, Weissleder R, et al. Magnetic nanoparticles and microNMR for diagnostic applications. Theranostics. 2012; 2: 55-65.

5. Ho DN, Kohler N, Sigdel A, Kalluri R, Morgan JR, Xu C, et al. Penetration of endothelial cell coated multicellular tumor spheroids by iron oxide nanoparticles. Theranostics. 2012; 2: 76-85.

6. Li M, Kim HS, Tian L, Yu MK, Jon S, Moon WK. Comparison of two ultrasmall superparamagnetic iron oxides on cytotoxicity and MR imaging of tumors. Theranostics. 2012; 2: 66-75.

7. Huang J, Zhong X, Wang L, Yang L, Mao H. Improving the magnetic resonance imaging contrast and detection methods with engineered magnetic nanoparticles. Theranostics. 2012; 2: 86-102.

8. Lee HY, Li Z, Chen K, Hsu AR, Xu C, Xie J, et al. PET/MRI dual-modality tumor imaging using arginine-glycine-aspartic (RGD)-conjugated radiolabeled iron oxide nanoparticles. J Nucl Med. 2008; 49: 1371-9.

9. Chen K, Xie J, Xu H, Behera D, Michalski MH, Biswal S, et al. Triblock copolymer coated iron oxide nanoparticle conjugate for tumor integrin targeting. Biomaterials. 2009; 30: 6912-9.

10. Xie J, Chen K, Huang J, Lee S, Wang J, Gao J, et al. PET/NIRF/MRI triple functional iron oxide nanoparticles. Biomaterials. 2010; 31: 3016-22.

11. Cai X, Yang F, Gu N. Applications of magnetic microbubbles for theranostics. Theranostics. 2012; 2: 103-112.

12. Zhao Q, Wang L, Cheng R, Mao l, Arnold RD, Howerth EW, et al. Magnetic nanoparticle-based hyperthermia for head \& neck cancer in mouse models. Theranostics. 2012; 2: 113-21. 